\title{
ACCESSIBILITY OF PROFESSIONAL HEALTH CARE (PRHC) IN GREATER BLOEMFONTEIN'
}

\author{
SJEJ van Vuuren and GW de Klerk
}

\begin{abstract}
The health of citizens is usually a priority in any society. In order to prevent/cure disease, people make use of various forms of care, ranging from lay care to professional health care ( $P R H C)$. Professional health care, however, is not equally accessible to all members of a society. This article attempts to indicate how factors such as costs, distance, consultation hours, attitude of medical personnel can result in PRHC being less accessible for some members and totally inaccessible for other members of a society. It is imperative that health care planners should once again review this issue in order to ensure that all South Africans are able to exercise their basic right to health care.
\end{abstract}

\section{OPSOMMING}

Gesondheid geniet meestal hoë prioriteit in enige samelewing. Mense maak dan ook van leke-tot professionele gesondheidsorg (PRGS) gebruik om siekte te bekamp. Professionele gesondheidsorg is egter nie vir almal ewe toeganklik nie. Hierdie artikel het ten doel om aan te toon dat faktore soos koste, afstand, konsultasietye, gesindheid van mediese personeel, ens daartoe lei dat PRGS vir sommmige groepe minder toeganklik en selfs ontoeganklik is. Indien gesondheidsorgbeplanners opnuut hiervan kennis neem, kan sodanig aangepas en/of verander word dat almal hul basiese reg tot gesondheidsorg in die Suid-Afrikaanse samelewing kan uitoefen.

\section{Research problem}

The accessibility of health care has a bearing on the extent to which (health) care services and facilities are open to the communities where they are rendered. Accessibility of PRHC becomes an issue when health care is not equally open to all or is open only to a segment of society as a result of restrictive factors. In addition, the accessibility/inaccessibility of health care is also determined by factors such as the ability/inability of users to pay for (health) care, membership/non-membership of health insurance schemes, the infrastructure by which health services are provided in the community, membership of specific groups, knowledge of available services, cultural preferences and aversions, obstacles of time and distance, etc. In the context of supply, accessibility/inaccessibility amounts to restrictive, discriminatory or exclusive measures (or the absence of such), and in the context of utilisation it denotes impecuniousness, lack of means, ignorance, etc. which promotes inaccessibility of care (Anyinam 1987:805-806; Benatar 1989; Van Rensburg \& Benatar 1993; Van Vuuren, De Klerk \& Van Rensburg 1993:8).
Against this background the article aims to highlight the health patterns of various groups and to illustrate the accessibility of PRHC in terms of the following factors:

- type of care initially approached for help

- frequency of utilization of PRHC services

- financing on PRHC services

- the cost of PRHC

- distance to PRHC service point

- attainability of PRHC service due to transport problems

- time elapsed before assistance was rendered by PRHC staff

- attitude of PRHC staff

- satisfaction with PRHC services

- convenience of times at which PRHC services are offered

- the perceived effect of PRHC on health

\section{RESEARCH METHODOLOGY}

In order to establish the accessibility of PRHC, interviews of approximately 45 minutes were conducted with 150 respondents from each of the white, coloured and black communities ${ }^{2}$ in the greater Bloemfontein area ${ }^{3}$. The interviews were conducted by six white students, eight coloured students and teachers and sixteen black students, all of whom were trained by the researcher. Interviews were conducted in Afrikaans, Enghsh or South Sotho - depending on the home language of the respondent. The multistage cluster sampling method was used to ensure random inclusion of 450 respondents. In the first phase of the survey, residential areas of Bloemfontein, Heidedal and Mangaung were randomly selected. In the second phase, dwelling units representing households were randomly selected by erf numbers. In order to give smaller areas an equal chance of inclusion in the survey, dwelling units were made proportional to the size of the residential area. Since the individual was to be the unit of analysis, the third phase involved the identification of a single respondent per residential unit on the basis of age. Although respondents of 18 years and older were selected by means of a matrix, age per sé was not taken into consideration in further analysis. The size of the separate samples was based on practical viability and the purposes of comparison. Since the survey was limited to residents of Bloemfontein, Heidedal and Mangaung, the results of the study are presented as relating solely to these communities.

\section{RESEARCH RESULTS}

Globally, PRHC is found mainly in areas of care such as solo practices, clinics and hospitals. Accessibility to these can be hampered by the way users pay for services; the cost of PRHC services; the distance from users' residences to PRHC points of service; the time elapsed before PRHC staff could help the user; the attitude of PRHC staff; satisfaction with PRHC services; times at which PRHC services are offered; the effect PRHC services have on health, etc. (Anyinam 1987:805-806; Coe 1978:413-414; Fosu 1989:398; Savage \& Benatar 1990:152). Accessibility of PRHC for the white, coloured and black communities in greater Bloemfontein will now be compared with reference to the mentioned variables. 
Table 1: Type of care initially approached for help

\begin{tabular}{lccccc|} 
Population group & chemist & $\begin{array}{c}\text { Area of care } \\
\text { private } \\
\text { practitioner }\end{array}$ & $\begin{array}{c}\text { Clinicl } \\
\text { Hospital }\end{array}$ & Other & Row Total \\
White & 31 & 108 & 4 & 6 & 149 \\
Colured & $21 \%$ & $72 \%$ & $3 \%$ & $4 \%$ & $100 \%$ \\
& 23 & 95 & 28 & 4 & 150 \\
Black & $15 \%$ & $63 \%$ & $19 \%$ & $3 \%$ & $100 \%$ \\
& 22 & 67 & 35 & 26 & 150 \\
Column Total & $15 \%$ & $45 \%$ & $23 \%$ & $17 \%$ & $100 \%$ \\
& 76 & 270 & 67 & 36 & 449 \\
$x^{2}=60,03055$ & $17 \%$ & $60 \%$ & $15 \%$ & $8 \%$ & $100 \%$ \\
& $\mathrm{dt}=6$ & $p=0,0000$ & & Uncertainty coefticient $=0,0667$ \\
\hline
\end{tabular}

Table 2: Frequency of utilisation of PRHC services

\begin{tabular}{|c|c|c|c|c|c|c|c|c|c|c|c|c|c|c|c|c|}
\hline \multirow{2}{*}{$\begin{array}{l}\text { Population } \\
\text { group }\end{array}$} & \multicolumn{3}{|c|}{$\begin{array}{l}\text { Medical prac- } \\
\text { tioner services }\end{array}$} & \multicolumn{5}{|c|}{ Clinic services } & \multicolumn{3}{|c|}{ Hospital services } & \multicolumn{4}{|c|}{ Total Row } & \multirow[b]{2}{*}{$s^{\text {TOT }}$} \\
\hline & $\underset{\text { times }}{2}$ & $\begin{array}{c}2-3 \\
\text { times }\end{array}$ & $\stackrel{>3}{\text { time }}$ & $\begin{array}{l}\text { TOT } \\
S\end{array}$ & $\stackrel{2}{\text { times }}$ & $\begin{array}{l}2-3 \\
\text { times }\end{array}$ & $\begin{array}{l}>3 \\
\text { times }\end{array}$ & TOT & $\underset{\text { times }}{<2}$ & $\begin{array}{l}2-3 \\
\text { times }\end{array}$ & $\begin{array}{l}>3 \\
\text { times }\end{array}$ & TOT & $\begin{array}{l}2 \\
\text { times }\end{array}$ & $\begin{array}{l}2-3 \\
\text { times }\end{array}$ & $\begin{array}{l}>3 \\
\text { times }\end{array}$ & \\
\hline White & 179 & 105 & 171 & 455 & 4 & 6 & 12 & 22 & 34 & 9 & 13 & 56 & 217 & 120 & 196 & 533 \\
\hline$\%$ & 39 & 23 & 38 & 100 & 18 & 27 & 55 & 100 & 61 & 16 & 23 & 100 & 41 & 22 & 37 & 100 \\
\hline Coloured & 65 & 45 & 52 & 162 & $\$ 1$ & 11 & 30 & 52 & 21 & 18 & 12 & 51 & 97 & 74 & 94 & 265 \\
\hline$\%$ & 40 & 28 & 32 & 100 & 21 & 21 & 58 & 100 & 41 & 35 & 24 & 100 & 37 & 28 & 35 & 100 \\
\hline Black & 126 & 89 & 96 & 311 & 16 & 16 & 48 & 80 & 72 & 32 & 76 & 180 & 214 & 137 & 220 & 571 \\
\hline$\%$ & 40 & 29 & 31 & 100 & 20 & 20 & 60 & 100 & 40 & 18 & 42 & 100 & 37 & 24 & 39 & 100 \\
\hline
\end{tabular}

Although, according to Andersen and Anderson (in Freeman, Levine \& Reeder 1979:387), differences within groups cannot be deduced from this, some indication of differences between groups with regard to the accessibility and eventual utilisation of PRHC can be achieved.

Investigation of the type of care which sick people initially approach (Table 1) reveals that white respondents' primary resource is the private practitioner. Proportionally more white respondents $(72 \%)$ than coloured $(63 \%)$ or black respondents (45\%) make use of this type of care. Because of their membership of medical funds, whites are in a better position than blacks or coloureds to afford the services of private practitioners. (Van Vuuren 1992:100-101) It is also noteworthy that more blacks (23\%) and coloureds (19\%) than whites (3\%) initially approach clinics and hospitals, while $17 \%$ of black respondents also indicated other family members, relations and friends as initial sources of help (Van Vuuren 1992:129).

Of the respondents who indicated that they had made use of PRHC services and facilities during the past year, $39 \%$ of whites, $40 \%$ of coloureds and $40 \%$ of blacks made use of some sor of medical practitioner service only once (Table 2). By contrast, 55\% of white respondents, $58 \%$ of coloured respondents and $60 \%$ of black respondents made use of clinic services more than three times during this period. As far as hospital services are concerned, the utilisation frequency of $61 \%$ for whites was less than twice during the past year. In the case of coloureds, $41 \%$ indicated that they had made use of hospital facilities only once during the year. For blacks, the figure was $40 \%$. However, the highest concentration for blacks occurs with a utilisation frequency of more than three times (42\%). This indicates that the various respondent groups use clinic services to a greater extent than they use medical practitioners and that black and coloured respondents make more use of hospital facilities than whites.

Among the chief reasons given by respondents for the utilisation of the various areas of care were, according to Van Vuuren (1992:126-127), the following:

- the patient's condition required hospital treatment;

- the specific service was perceived as most suitable;

- the service point was easily accessible;

- a doctor had been recommended by others;

- the service given by the doctor was satisfactory;

- clinic services are cheaper than general practitioners;

- the specific practitioner had been the patient's family doctor for years, and

- private practitioners give individual care, which was preferred.

\section{Financing of PRHC services (Table 3)}

The data in Table 3 indicate that white respondents generally make use of medical schemes to pay for the services of medical

\begin{tabular}{|c|c|c|c|c|c|c|c|c|c|c|c|c|c|c|c|c|c|c|c|c|}
\hline \multirow[t]{2}{*}{$\begin{array}{l}\text { Population } \\
\text { group }\end{array}$} & \multicolumn{5}{|c|}{$\begin{array}{l}\text { Medical prac- } \\
\text { tioner services }\end{array}$} & \multicolumn{5}{|c|}{ Clinic services } & \multicolumn{5}{|c|}{ Hospital services } & \multicolumn{5}{|c|}{ Total Row } \\
\hline & $\begin{array}{l}\text { Own } \\
\text { poc- }\end{array}$ & $\begin{array}{l}\text { Med } \\
\text { fund }\end{array}$ & Free & Other & Tot & $\begin{array}{l}\text { Own } \\
\text { poc. }\end{array}$ & $\begin{array}{l}\text { Med } \\
\text { hund }\end{array}$ & Free & Other & Tot & $\begin{array}{l}\text { Own } \\
\text { poc- }\end{array}$ & $\begin{array}{l}\text { Med } \\
\text { fund }\end{array}$ & Free & Other & Tot & $\begin{array}{l}\text { Own } \\
\text { poc. }\end{array}$ & $\begin{array}{l}\text { Med } \\
\text { fund }\end{array}$ & Free & Other & Tot \\
\hline White & 52 & 392 & 7 & 64 & 457 & $\cdot$ & 7 & 15 & - & 22 & 6 & 44 & 5 & 1 & 56 & 58 & 443 & 27 & 7 & 535 \\
\hline$\%$ & 11 & 86 & 2 & 1 & 100 & $\cdot$ & 32 & 68 & - & 100 & 11 & 79 & 8 & 2 & 100 & 11 & 83 & 5 & 1 & 100 \\
\hline Coloured & 84 & 78 & 4 & - & 166 & 12 & - & 41 & - & 53 & 34 & 9 & 5 & 1 & 49 & 130 & 87 & 50 & 1 & 268 \\
\hline$\%$ & 51 & 47 & 2 & - & 100 & 23 & $\cdot$ & $\pi$ & - & 100 & 69 & 19 & 10 & 2 & 100 & 49 & 32 & 18,5 & 0,5 & 100 \\
\hline Black & 180 & 63 & 11 & 59 & 313 & 15 & 1 & 60 & 4 & 80 & 101 & 9 & 28 & 41 & 179 & 296 & 73 & 99 & 104 & 572 \\
\hline$\%$ & 57 & 20 & 4 & 19 & 100 & 19 & 1 & 75 & 5 & 100 & 56 & 5 & 16 & 23 & 100 & 52 & 13 & 17 & 18 & 100 \\
\hline
\end{tabular}


Table 4: The cost of PRHC services

\begin{tabular}{|c|c|c|c|c|c|c|c|c|c|c|c|c|}
\hline \multirow[t]{2}{*}{$\begin{array}{l}\text { Population } \\
\text { group }\end{array}$} & \multicolumn{3}{|c|}{$\begin{array}{l}\text { Medical practi- } \\
\text { tioner services }\end{array}$} & \multicolumn{3}{|c|}{ Clinic services } & \multicolumn{3}{|c|}{ Hospital services } & \multicolumn{3}{|c|}{ Row total } \\
\hline & $\begin{array}{l}\text { Too } \\
\text { high }\end{array}$ & $\begin{array}{l}\text { Reason- } \\
\text { able }\end{array}$ & TOT & $\begin{array}{l}\text { To } \\
\text { bigh }\end{array}$ & $\begin{array}{l}\text { Reas } \\
\text { able }\end{array}$ & TOT & $\begin{array}{l}\text { Too } \\
\text { high }\end{array}$ & $\begin{array}{l}\text { Reas } \\
\text { able }\end{array}$ & TOT & $\begin{array}{l}\text { Too } \\
\text { bigh }\end{array}$ & $\begin{array}{l}\text { Reas } \\
\text { able }\end{array}$ & -TOT \\
\hline $\begin{array}{l}\text { While } \\
\%\end{array}$ & $\begin{array}{r}143 \\
32\end{array}$ & $\begin{array}{r}300 \\
68\end{array}$ & $\begin{array}{l}443 \\
100\end{array}$ & $\begin{array}{r}7 \\
78\end{array}$ & $\begin{array}{r}2 \\
22\end{array}$ & $\begin{array}{r}9 \\
100\end{array}$ & $\begin{array}{l}4 \\
8\end{array}$ & $\begin{array}{l}44 \\
92\end{array}$ & $\begin{array}{r}48 \\
100\end{array}$ & $\begin{array}{r}154 \\
31\end{array}$ & $\begin{array}{r}346 \\
69\end{array}$ & $\begin{array}{l}500 \\
100\end{array}$ \\
\hline $\begin{array}{l}\text { Coloured } \\
\%\end{array}$ & $\begin{array}{l}47 \\
31\end{array}$ & $\begin{array}{r}105 \\
69\end{array}$ & $\begin{array}{l}152 \\
100\end{array}$ & $\begin{array}{r}2 \\
22\end{array}$ & $\begin{array}{r}7 \\
78\end{array}$ & $\begin{array}{r}9 \\
100\end{array}$ & $\begin{array}{l}10 \\
24\end{array}$ & $\begin{array}{l}31 \\
76\end{array}$ & $\begin{array}{r}41 \\
100\end{array}$ & $\begin{array}{l}59 \\
29\end{array}$ & $\begin{array}{r}143 \\
71\end{array}$ & $\begin{array}{l}202 \\
100\end{array}$ \\
\hline $\begin{array}{l}\text { Black } \\
\%\end{array}$ & $\begin{array}{l}97 \\
34\end{array}$ & $\begin{array}{r}192 \\
66\end{array}$ & $\begin{array}{l}289 \\
100\end{array}$ & $\begin{array}{r}7 \\
32\end{array}$ & $\begin{array}{l}15 \\
68\end{array}$ & $\begin{array}{r}22 \\
100\end{array}$ & $\begin{array}{l}33 \\
21\end{array}$ & $\begin{array}{r}126 \\
79\end{array}$ & $\begin{array}{l}159 \\
100\end{array}$ & $\begin{array}{r}137 \\
29\end{array}$ & $\begin{array}{r}333 \\
71\end{array}$ & $\begin{array}{l}470 \\
100\end{array}$ \\
\hline
\end{tabular}

Table 5: Distance from residence to PRHC point of service

\begin{tabular}{|c|c|c|c|c|c|c|c|c|c|c|c|c|}
\hline \multirow[t]{2}{*}{$\begin{array}{l}\text { Population } \\
\text { group }\end{array}$} & \multicolumn{3}{|c|}{$\begin{array}{l}\text { Medical practi- } \\
\text { lioner services }\end{array}$} & \multicolumn{3}{|c|}{ Clinic services } & \multicolumn{3}{|c|}{ Hospital services } & \multicolumn{3}{|c|}{ Row total } \\
\hline & $\begin{array}{l}\text { Far } \\
\text { away }\end{array}$ & $\begin{array}{l}\text { Near } \\
\text { by }\end{array}$ & TOT & $\begin{array}{l}\text { Far } \\
\text { away }\end{array}$ & $\begin{array}{l}\text { Near } \\
\text { by }\end{array}$ & TOT & $\begin{array}{l}\text { Far } \\
\text { away }\end{array}$ & $\begin{array}{l}\text { Near } \\
\text { by }\end{array}$ & TOT & $\begin{array}{l}\text { Far } \\
\text { away }\end{array}$ & $\begin{array}{l}\text { Near } \\
\text { by }\end{array}$ & TOT \\
\hline $\begin{array}{l}\text { White } \\
\%\end{array}$ & $\begin{array}{r}100 \\
22\end{array}$ & $\begin{array}{r}356 \\
78\end{array}$ & $\begin{array}{l}456 \\
100\end{array}$ & $\frac{5}{23}$ & $\begin{array}{l}17 \\
77\end{array}$ & $\frac{22}{100}$ & $\begin{array}{l}27 \\
49\end{array}$ & $\begin{array}{l}28 \\
51\end{array}$ & $\begin{array}{r}55 \\
100\end{array}$ & $\begin{array}{r}132 \\
25\end{array}$ & $\begin{array}{r}401 \\
75\end{array}$ & $\begin{array}{l}533 \\
100\end{array}$ \\
\hline $\begin{array}{l}\text { Coloured } \\
\%\end{array}$ & $\begin{array}{l}62 \\
37\end{array}$ & $\begin{array}{r}104 \\
63\end{array}$ & $\begin{array}{l}166 \\
100\end{array}$ & $\begin{array}{r}7 \\
13\end{array}$ & $\begin{array}{l}46 \\
87\end{array}$ & $\begin{array}{r}53 \\
100\end{array}$ & $\begin{array}{l}13 \\
27\end{array}$ & $\begin{array}{l}36 \\
73\end{array}$ & $\begin{array}{r}49 \\
100\end{array}$ & $\begin{array}{l}82 \\
31\end{array}$ & $\begin{array}{r}186 \\
69\end{array}$ & $\begin{array}{l}268 \\
100\end{array}$ \\
\hline $\begin{array}{l}\text { Black } \\
\%\end{array}$ & $\begin{array}{r}165 \\
53\end{array}$ & $\begin{array}{r}147 \\
47\end{array}$ & $\begin{array}{l}312 \\
100\end{array}$ & $\begin{array}{l}29 \\
37\end{array}$ & $\begin{array}{l}50 \\
63\end{array}$ & $\begin{array}{r}79 \\
100\end{array}$ & $\begin{array}{c}104 \\
59\end{array}$ & $\begin{array}{l}71 \\
41\end{array}$ & $\begin{array}{l}175 \\
100\end{array}$ & $\begin{array}{r}298 \\
53\end{array}$ & $\begin{array}{r}268 \\
47\end{array}$ & $\begin{array}{l}566 \\
100\end{array}$ \\
\hline
\end{tabular}

practitioners. By contrast, black and coloured respondents generally have to pay personally for the services of medical practitioners because fewer blacks and coloureds than whites are members of medical aid schemes. (Van Vuuren 1992:100-101). The fact that coloured and black respondents do not enjoy the same degree of help from medical aid schemes in the payment of medical expenses as whites may imply that coloureds and blacks make less use, or no use at all of expensive health services, merely because these services are not affordable and therefore less accessible to these groups (Van Vuuren 1992:129-130).

\section{The cost of PRHC services (Table 4)}

Varying responses were obtained concerning the amount payable for PRHC services (Table 4). Although all three groups initially indicated that the fees payable for the services of medical practitioners $(68 \%, 69 \%, 66 \%$, respectively) and hospitals $(92 \%, 76 \%, 79 \%)$ were reasonable, both white (78\%) and black (32\%) respondents felt that the fees payable for clinic services were too high. The clinic services referred to here are probably those black 34\%) nevertheless felt that tariffs for this type of service were too high. This implies that, for financial reasons, PRHC is inaccessible for a third of the group under investigation (Van Vuuren 1992:131-132).

Distance from residence to PRHC point of service (Table 5)

In order to establish whether distance was a factor inhibiting respondents from making use of required PRHC services, they were asked whether their usual PRHC service point was near or far away from their residence. According to the responses in Table 5, white respondents $(78 \%)$ indicated that medical practitioners' points of service were near to their residences. By contrast, less coloured (63\%) and black (47\%) respondents stated that they hived within reach of a PRHC point of service. This indicates that, in terms of distance, the services of medical practitioners are less accessible for coloureds and blacks than for whites

When one considers the actual distances which users must travel to points of service for clinics and hospitals, one can theoretically argue that with modem transportation means service points are within reach. Nevertheless, more than a third of black respondents (37\%) said that they lived far from these points of service. In the case of hospital service points, $49 \%$ of whites and $59 \%$ of blacks reported that hospital points of service were far away. In terms of distance, thus, clinic and hospital services are relatively inaccessible for certain groups (Van Vuuren 1992:132-133).

\section{Attainability of PRHC arising from transport problems (Table 6)}

In connection with the previous point, and in order to identify possible transport problems, respondents were asked whether they found it easy or difficult to reach their usual PRHC point of service. Although relatively high percentages of responses $(93 \%, 88 \%, 70 \%$, respectively) indicated that it is easy for the group under investigation as a whole to reach the various PRHC points of service, it must be than services available in municipal clinics. Although two-thirds of respondents considered the cost of services of medical the various groups (white $32 \%$, coloured $31 \%$,

Table 6: Attainability of PRHC arising from transport problems

\begin{tabular}{|c|c|c|c|c|c|c|c|c|c|c|c|c|}
\hline \multirow[t]{2}{*}{$\begin{array}{l}\text { Population } \\
\text { group }\end{array}$} & \multicolumn{3}{|c|}{$\begin{array}{l}\text { Medical practi- } \\
\text { tioner services }\end{array}$} & \multicolumn{3}{|c|}{ Cinic services } & \multicolumn{3}{|c|}{ Hospital services } & \multicolumn{3}{|c|}{ Row total } \\
\hline & Eary & $\begin{array}{l}\text { Diff- } \\
\text { cult }\end{array}$ & TOT & Easy & $\begin{array}{l}\text { Difts- } \\
\text { cult }\end{array}$ & TOT & Easy & $\begin{array}{l}\text { Difr- } \\
\text { cull }\end{array}$ & TOT & Easy & $\begin{array}{l}\text { Difl- } \\
\text { cult }\end{array}$ & TOT \\
\hline $\begin{array}{l}\text { White } \\
\%\end{array}$ & $\begin{array}{r}431 \\
95\end{array}$ & $\begin{array}{r}25 \\
5\end{array}$ & $\begin{array}{l}456 \\
100\end{array}$ & $\begin{array}{l}16 \\
73\end{array}$ & $\begin{array}{r}6 \\
27\end{array}$ & $\frac{22}{100}$ & $\begin{array}{l}47 \\
85\end{array}$ & $\begin{array}{r}8 \\
15\end{array}$ & $\begin{array}{r}55 \\
100\end{array}$ & $\begin{array}{r}494 \\
93\end{array}$ & $\begin{array}{r}39 \\
7\end{array}$ & $\begin{array}{l}533 \\
100\end{array}$ \\
\hline $\begin{array}{l}\text { Coloured } \\
\%\end{array}$ & $\begin{array}{r}143 \\
86\end{array}$ & $\begin{array}{l}23 \\
14\end{array}$ & $\begin{array}{l}166 \\
100\end{array}$ & 94 & $\begin{array}{l}3 \\
6\end{array}$ & $\begin{array}{r}52 \\
100\end{array}$ & $\begin{array}{l}42 \\
86\end{array}$ & $\begin{array}{r}7 \\
14\end{array}$ & $\begin{array}{r}49 \\
100\end{array}$ & $\begin{array}{r}234 \\
88\end{array}$ & $\begin{array}{l}33 \\
12\end{array}$ & $\begin{array}{l}267 \\
100\end{array}$ \\
\hline $\begin{array}{l}\text { Black } \\
\text { \% }\end{array}$ & $\begin{array}{r}213 \\
69\end{array}$ & $\begin{array}{l}97 \\
31\end{array}$ & $\begin{array}{l}310 \\
100\end{array}$ & $\begin{array}{l}62 \\
79\end{array}$ & $\begin{array}{l}16 \\
21\end{array}$ & $\begin{array}{r}78 \\
100\end{array}$ & $\begin{array}{r}117 \\
67\end{array}$ & $\begin{array}{l}58 \\
33\end{array}$ & $\begin{array}{l}175 \\
100\end{array}$ & $\begin{array}{r}392 \\
70\end{array}$ & $\begin{array}{r}171 \\
30\end{array}$ & $\begin{array}{l}563 \\
100\end{array}$ \\
\hline
\end{tabular}


Table 7: Time elapsed before PAHC staff could render assistance

\begin{tabular}{|c|c|c|c|c|c|c|c|c|c|c|c|c|}
\hline \multirow[t]{2}{*}{$\begin{array}{l}\text { Population } \\
\text { group }\end{array}$} & \multicolumn{3}{|c|}{$\begin{array}{l}\text { Medical practi- } \\
\text { tioner staff }\end{array}$} & \multicolumn{3}{|c|}{ Clinic staff } & \multicolumn{3}{|c|}{ Hospital staff } & \multicolumn{3}{|c|}{ Row total } \\
\hline & $\begin{array}{l}\text { Assis- } \\
\text { ted } \\
\text { quickly }\end{array}$ & $\begin{array}{l}\text { Wait } \\
\text { long }\end{array}$ & TOT & $\begin{array}{l}\text { Assis- } \\
\text { led } \\
\text { quickly }\end{array}$ & $\begin{array}{l}\text { Waited } \\
\text { long }\end{array}$ & TOT & $\begin{array}{l}\text { Assis- } \\
\text { ted } \\
\text { quickly }\end{array}$ & $\begin{array}{l}\text { Waited } \\
\text { long }\end{array}$ & TOT & $\begin{array}{l}\text { Assis- } \\
\text { ted } \\
\text { quickly }\end{array}$ & $\begin{array}{l}\text { Waited } \\
\text { long }\end{array}$ & TOT \\
\hline $\begin{array}{l}\text { White } \\
\%\end{array}$ & $\begin{array}{r}406 \\
89\end{array}$ & $\begin{array}{l}49 \\
11\end{array}$ & $\begin{array}{l}455 \\
100\end{array}$ & $\begin{array}{l}21 \\
95\end{array}$ & $\begin{array}{l}1 \\
5\end{array}$ & $\begin{array}{r}22 \\
100\end{array}$ & $\begin{array}{l}50 \\
91\end{array}$ & $\begin{array}{l}5 \\
9\end{array}$ & $\begin{array}{r}55 \\
100\end{array}$ & $\begin{array}{r}477 \\
90\end{array}$ & $\begin{array}{l}55 \\
10\end{array}$ & $\begin{array}{l}532 \\
100\end{array}$ \\
\hline $\begin{array}{l}\text { Coloured } \\
\%\end{array}$ & $\begin{array}{r}124 \\
75\end{array}$ & $\begin{array}{l}41 \\
25\end{array}$ & $\begin{array}{l}165 \\
100\end{array}$ & $\begin{array}{l}38 \\
73\end{array}$ & $\begin{array}{l}14 \\
27\end{array}$ & $\begin{array}{r}52 \\
100\end{array}$ & $\begin{array}{l}21 \\
43\end{array}$ & $\begin{array}{l}28 \\
57\end{array}$ & $\begin{array}{r}49 \\
100\end{array}$ & $\begin{array}{r}183 \\
69\end{array}$ & $\begin{array}{l}83 \\
31\end{array}$ & $\begin{array}{l}266 \\
100\end{array}$ \\
\hline $\begin{array}{l}\text { Black } \\
\%\end{array}$ & $\begin{array}{r}205 \\
66\end{array}$ & $\begin{array}{r}104 \\
34\end{array}$ & $\begin{array}{l}309 \\
100\end{array}$ & $\begin{array}{l}48 \\
62\end{array}$ & $\begin{array}{l}30 \\
38\end{array}$ & $\begin{array}{r}78 \\
100\end{array}$ & $\begin{array}{l}79 \\
46\end{array}$ & $\begin{array}{l}93 \\
54\end{array}$ & $\begin{array}{l}172 \\
100\end{array}$ & $\begin{array}{r}332 \\
59\end{array}$ & 227 & $\begin{array}{l}559 \\
100\end{array}$ \\
\hline
\end{tabular}

Table 8: Attitude of PRHC staff

\begin{tabular}{|c|c|c|c|c|c|c|c|c|c|c|c|c|c|c|c|}
\hline \multirow{3}{*}{$\begin{array}{l}\text { Population } \\
\text { group } \\
\\
\text { White } \\
\%\end{array}$} & \multicolumn{3}{|c|}{$\begin{array}{l}\text { Medical practi- } \\
\text { toper staff }\end{array}$} & \multicolumn{4}{|c|}{ Clinic staff } & \multicolumn{4}{|c|}{$\begin{array}{c}\text { Hospital staff } \\
.\end{array}$} & \multicolumn{4}{|c|}{ Row total } \\
\hline & $\begin{array}{l}\text { Prien } \\
\text { iy }\end{array}$ & New & $\begin{array}{l}\text { Un- TOT } \\
\text { friend- } \\
\text { hy }\end{array}$ & $\begin{array}{l}\text { Priend- } \\
\text { by }\end{array}$ & $\begin{array}{c}\text { Nev- } \\
\text { onal }\end{array}$ & $\begin{array}{l}\text { Un- } \\
\text { fried } \\
\text { hy }\end{array}$ & TOT & $\begin{array}{l}\text { Priend- } \\
\text { iy }\end{array}$ & $\begin{array}{l}\text { Nev- } \\
\text { tral }\end{array}$ & $\begin{array}{l}\text { Ua- } \\
\text { trienot } \\
\text { it }\end{array}$ & TOT & $\begin{array}{l}\text { Friend- } \\
h\end{array}$ & $\begin{array}{l}\text { Nev- } \\
\text { tral }\end{array}$ & $\begin{array}{l}\text { Un- } \\
\text { friend } \\
\text { by }\end{array}$ & ${ }^{\text {TOT }}$ \\
\hline & $\begin{array}{r}432 \\
95\end{array}$ & $\frac{22}{5}$ & $\begin{array}{l}\cdot 454 \\
\cdot \quad 100\end{array}$ & $\begin{array}{l}16 \\
73\end{array}$ & $\begin{array}{r}5 \\
23\end{array}$ & $\begin{array}{l}1 \\
4\end{array}$ & $\begin{array}{r}22 \\
100\end{array}$ & $\begin{array}{l}53 \\
96\end{array}$ & 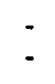 & $\begin{array}{l}2 \\
41\end{array}$ & $\begin{array}{r}55 \\
100\end{array}$ & $\begin{array}{r}501 \\
94\end{array}$ & $\begin{array}{r}27 \\
5\end{array}$ & $\begin{array}{l}3 \\
1\end{array}$ & $\begin{array}{l}531 \\
100\end{array}$ \\
\hline $\begin{array}{l}\text { Coloured } \\
\%\end{array}$ & $\begin{array}{r}154 \\
94\end{array}$ & $\begin{array}{l}6 \\
4\end{array}$ & $\begin{array}{ll}4 & 164 \\
2 & 100\end{array}$ & $\begin{array}{l}50 \\
94\end{array}$ & $\begin{array}{l}2 \\
4\end{array}$ & $\begin{array}{l}1 \\
2\end{array}$ & $\begin{array}{r}53 \\
100\end{array}$ & $\begin{array}{l}37 \\
76\end{array}$ & $\begin{array}{l}4 \\
8\end{array}$ & $\begin{array}{r}8 \\
16\end{array}$ & $\begin{array}{r}49 \\
100\end{array}$ & $\begin{array}{r}241 \\
91\end{array}$ & 12 & $\begin{array}{r}13 \\
5\end{array}$ & $\begin{array}{l}266 \\
100\end{array}$ \\
\hline $\begin{array}{l}\text { Black } \\
\%\end{array}$ & $\begin{array}{r}282 \\
91\end{array}$ & $\begin{array}{c}25 \\
8\end{array}$ & $\begin{array}{ll}3 & 310 \\
1 & 100\end{array}$ & $\begin{array}{l}55 \\
71\end{array}$ & $\begin{array}{l}18 \\
23\end{array}$ & $\begin{array}{l}5 \\
6\end{array}$ & $\begin{array}{r}78 \\
100\end{array}$ & $\begin{array}{r}123 \\
72\end{array}$ & $\begin{array}{l}40 \\
23\end{array}$ & $\begin{array}{l}8 \\
5\end{array}$ & $\begin{array}{l}171 \\
100\end{array}$ & $\begin{array}{r}460 \\
82\end{array}$ & $\begin{array}{l}83 \\
15\end{array}$ & $\begin{array}{r}16 \\
3 .\end{array}$ & $\begin{array}{r}559 \\
.100\end{array}$ \\
\hline
\end{tabular}

noted that some white respondents found it difficult to reach clinic points of service $(27 \%)$; that coloured respondents reported similarly with regard to both medical practitioners $(14 \%)$ and hospital points of service (14\%), and that black respondents had difficulty in reaching medical practitioners $(31 \%)$ as well as clinics (21\%) and hospital points of service (33\%) (Table 6). From these figures one can deduce that PRHC services are less accessible to black respondents than to coloured and white respondents. Reasons given by respondents for their difficulty in reaching PRHC points of service included problems with transport and lack of funds to pay for available transport. A single respondent identified the unsafe conditions in the residential area as an inhibiting factor in reaching a PRHC point of service (Van Vuuren 1992:132-133).

\section{Time elapsed before PRHC staff could} render assistance (Table 7 )

Although the responses in Table 7 indicate that, as a whole, the group under investigation reported that they had been assisted quickly at and coloureds more quickly than blacks. While white respondents were quickly assisted at all three types of service points, coloureds (57\%) and blacks (54\%) reported a long wait before hospital staff could attend to them. In addition, ${ }^{\circ}$ coloured and black respondents also reported waiting for the services of clinics $(27 \%, 38 \%)$ and medical practitioners $(25 \%, 34 \%)$. When making use of PRHC services, thus, the time elapsed before being treated is more of an inhibiting factor for black and coloured respondents than for white respondents. The main reasons given by respondents for the need to wait include according to Van Vuuren (1992:134-135):

- a shortage of medical personnel;

- the slowness of doctors and nurses;

- time-consuming administrative procedures, and

- large numbers of patients requiring treatment.

\section{Attitude of PRHC staff (Table 8)}

As a whole, the responses in Table 8 indicate that the group under investigation - white $(94 \%)$, coloured $(91 \%)$ and black $(82 \%)$. perceived staff at the points of service which they used as friendly to very friendly. Nevertheless, it is clear that some white (23\%) and black respondents (23\%) reported their opinion of the friendliness of clinic staff as neutral and that black respondents (23\%) were similarly neutral about the friendliness of hospital staff. In addition, responses from coloured respondents $(16 \%)$ indicated that they found hospital staff unfriendly - an perception which may well inhibit coloured respondents from using hospital services. Most white $(95 \%)$, coloured $(94 \%)$ and black (91\%) respondents found that medical practitioners treated them in a friendly manner (Van Vuuren 1992:135-136)

$69 \%, 59 \%$ ), it is noteworthy that responses in the "waited long" category increase from whites $(10 \%)$ to coloureds $(31 \%)$ and to blacks (41\%). This means that white respondents were assisted more quickly than coloureds,

Satisfaction with PRHC services

(Table 9)

Table 9 Satisfaction with PRHC services

\begin{tabular}{|c|c|c|c|c|c|c|c|c|c|c|c|c|}
\hline \multirow{2}{*}{$\begin{array}{l}\text { Population } \\
\text { goup } \\
\text {. }\end{array}$} & \multicolumn{3}{|c|}{$\begin{array}{l}\text { Medical practi- } \\
\text { tooer services }\end{array}$} & \multicolumn{3}{|c|}{ Clinic services } & \multicolumn{3}{|c|}{ Hospital services } & \multicolumn{3}{|c|}{ Row total } \\
\hline & $\begin{array}{l}\text { Satis- } \\
\text { Ged }\end{array}$ & $\begin{array}{l}\text { Unsa- } \\
\text { tis- } \\
\text { fied }\end{array}$ & TOT & $\begin{array}{l}\text { Satis- } \\
\text { fied }\end{array}$ & $\begin{array}{l}\text { Unsa- } \\
\text { tis- } \\
\text { fied }\end{array}$ & TOT & $\begin{array}{l}\text { Satis- } \\
\text { fied }\end{array}$ & $\begin{array}{l}\text { Unsa- } \\
\text { lis- } \\
\text { fied }\end{array}$ & TOT & $\begin{array}{l}\text { Satis- } \\
\text { fied }\end{array}$ & $\begin{array}{l}\text { Unss- } \\
\text { tis- } \\
\text { fied }\end{array}$ & TOT \\
\hline $\begin{array}{l}\text { White } \\
\%\end{array}$ & $\begin{array}{r}451 \\
99\end{array}$ & $\begin{array}{l}4 \\
1\end{array}$ & $\begin{array}{l}455 \\
100\end{array}$ & $\begin{array}{l}20 \\
91\end{array}$ & $\begin{array}{l}2 \\
9\end{array}$ & $\frac{22}{100}$ & $\begin{array}{l}52 \\
95\end{array}$ & $\begin{array}{l}3 \\
5\end{array}$ & $\begin{array}{r}55 \\
100\end{array}$ & $\begin{array}{r}523 \\
98\end{array}$ & $\begin{array}{l}9 \\
2\end{array}$ & $\begin{array}{l}532 \\
100\end{array}$ \\
\hline $\begin{array}{l}\text { Coloured } \\
\%\end{array}$ & $\begin{array}{l}157 \\
95\end{array}$ & $\begin{array}{l}9 \\
5\end{array}$ & $\begin{array}{l}166 \\
100\end{array}$ & $\begin{array}{l}49 \\
96\end{array}$ & $\begin{array}{l}2 \\
4\end{array}$ & $\begin{array}{r}51 \\
100\end{array}$ & $\begin{array}{l}39 \\
81\end{array}$ & $\begin{array}{r}9 \\
19\end{array}$ & $\begin{array}{r}48 \\
100\end{array}$ & $\begin{array}{r}245 \\
92\end{array}$ & $\begin{array}{r}20 \\
8\end{array}$ & $\begin{array}{l}265 \\
100\end{array}$ \\
\hline $\begin{array}{l}\text { Black } \\
\%\end{array}$ & $\begin{array}{r}292 \\
96\end{array}$ & $\begin{array}{r}13 \\
4\end{array}$ & $\begin{array}{l}305 \\
100\end{array}$ & $\begin{array}{l}71 \\
95\end{array}$ & $\begin{array}{l}4 \\
5\end{array}$ & $\begin{array}{r}75 \\
100\end{array}$ & $\begin{array}{r}159 \\
92\end{array}$ & $\begin{array}{r}14 \\
8\end{array}$ & $\begin{array}{l}173 \\
100\end{array}$ & 522 & $\begin{array}{r}31 \\
6\end{array}$ & $\begin{array}{l}553 \\
100\end{array}$ \\
\hline
\end{tabular}


According to Table 9, all three population groups $(98 \%, 92 \%, 94 \%)$ indicated that they were satisfied with the PRHC services which they had received. However, $19 \%$ of coloured and $8 \%$ of black respondents stated that they were dissatisfied with hospital services. A few white respondents $(9 \%)$ reported dissatisfaction with clinic services. The complaints of the dissatisfied respondents, regardless of type of care, related, according to Van Vuuren (1992:137-138), mainly to:

- the behaviour of medical personnel (particularly black nurses towards coloured patients);

- incorrect diagnoses by personnel;

- incorrect medication given, and

- doctors' lack of involvement with patients.

The fact that coloureds were more dissatisfied than either blacks or whites with the PRHC services which should provide for their health care requirements is probably due to the marginal position of coloured people in the community. It is likely that this dissatisfaction may play a role in the utilisation of PRHC services and facilities by this group.

Convenience of times at which PRHC services are offered (Table 10)

Although the responses of all three groups white $(97 \%)$, coloured $(82 \%)$ and black $(92 \%)$ - identified the times at which the various types of PRHC services are offered as generally convenient, a considerable proportion of coloured respondents characterised the times at which the services of medical practitioners $(18 \%)$, clinics $(17 \%)$ and hospitals $(21 \%)$ could be used as inconvenient. This implies that the timing of PRHC services is probably a greater inhibiting factor for coloureds than for blacks or whites (Table 10) (Van Vuuren 1992:138-139).

\section{The perceived effect of PRHC on health (Table 11)}

In order to establish whether the use of PRHC services had a perceived effect on respondents' health, they were asked whether their health had improved, deteriorated, or remained the same after treatment. Although

Table 10 Convenience of times at which PRHC services are offered

\begin{tabular}{|c|c|c|c|c|c|c|c|c|c|c|c|c|}
\hline \multirow[t]{2}{*}{$\begin{array}{l}\text { Population } \\
\text { goup }\end{array}$} & \multicolumn{3}{|c|}{$\begin{array}{l}\text { Medical practi- } \\
\text { tioner services }\end{array}$} & \multicolumn{3}{|c|}{ Clinic services } & \multicolumn{3}{|c|}{ Hospital services } & \multicolumn{3}{|c|}{ Row total } \\
\hline & $\begin{array}{l}\text { Conve- } \\
\text { nieat }\end{array}$ & $\begin{array}{l}\text { Inco } \\
\text { venie }\end{array}$ & TOT & $\begin{array}{l}\text { Conve- } \\
\text { nient }\end{array}$ & $\begin{array}{l}\text { Incon- } \\
\text { venient }\end{array}$ & TOT & $\begin{array}{l}\text { Conve- } \\
\text { nient }\end{array}$ & $\begin{array}{l}\text { Incon- } \\
\text { venien }\end{array}$ & TOT & $\begin{array}{l}\text { Conve- } \\
\text { nient }\end{array}$ & $\begin{array}{l}\text { locon- } \\
\text { venient }\end{array}$ & TOT \\
\hline $\begin{array}{l}\text { White } \\
\%\end{array}$ & $\begin{array}{r}444 \\
98\end{array}$ & $\begin{array}{r}11 \\
2\end{array}$ & $\begin{array}{l}455 \\
100\end{array}$ & $\begin{array}{l}20 \\
91\end{array}$ & $\begin{array}{l}2 \\
9\end{array}$ & $\frac{22}{100}$ & $\begin{array}{l}53 \\
98\end{array}$ & $\begin{array}{l}1 \\
2\end{array}$ & $\begin{array}{r}54 \\
100\end{array}$ & $\begin{array}{r}517 \\
97\end{array}$ & $\begin{array}{r}14 \\
3\end{array}$ & $\begin{array}{l}531 \\
100\end{array}$ \\
\hline $\begin{array}{l}\text { Coloured } \\
\%\end{array}$ & $\begin{array}{l}133 \\
82\end{array}$ & $\begin{array}{l}29 \\
18\end{array}$ & $\begin{array}{l}162 \\
100\end{array}$ & $\begin{array}{l}44 \\
83\end{array}$ & $\begin{array}{r}9 \\
17\end{array}$ & $\begin{array}{r}53 \\
100\end{array}$ & $\begin{array}{l}38 \\
79\end{array}$ & $\begin{array}{l}10 \\
21\end{array}$ & $\begin{array}{r}48 \\
100\end{array}$ & $\begin{array}{r}215 \\
82\end{array}$ & $\begin{array}{l}48 \\
18\end{array}$ & $\begin{array}{l}263 \\
100\end{array}$ \\
\hline $\begin{array}{l}\text { Black } \\
\%\end{array}$ & $\begin{array}{r}274 \\
92\end{array}$ & $\begin{array}{r}23 \\
8\end{array}$ & $\begin{array}{l}297 \\
100\end{array}$ & $\begin{array}{l}65 \\
89\end{array}$ & $\begin{array}{r}8 \\
11\end{array}$ & $\begin{array}{r}73 \\
100\end{array}$ & $\begin{array}{r}139 \\
93\end{array}$ & $\begin{array}{r}10 \\
7\end{array}$ & $\begin{array}{l}149 \\
100\end{array}$ & $\begin{array}{r}478 \\
92\end{array}$ & $\begin{array}{r}41 \\
8\end{array}$ & $\begin{array}{l}519 \\
100\end{array}$ \\
\hline
\end{tabular}

Table 11: The perceived effect of PRHC on health

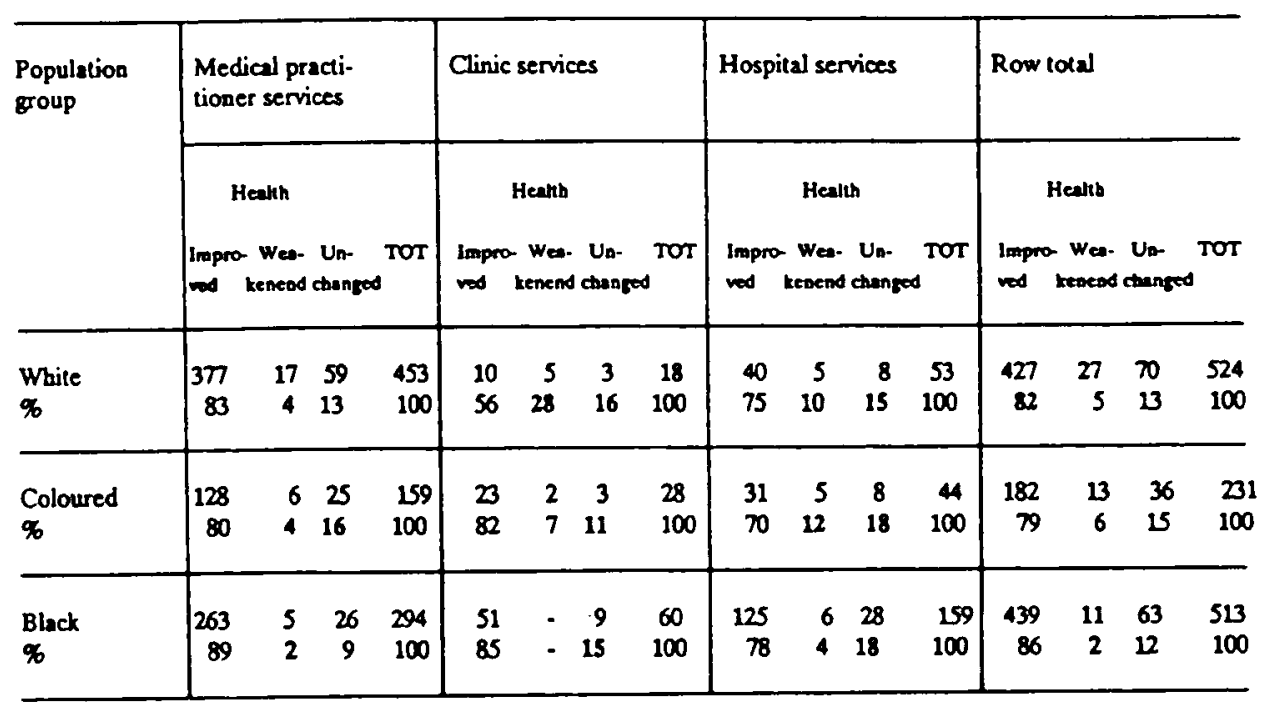

most responses (Table 11) indicated that respondents' health had improved after treatment at a PRHC point of service, a considerable proportion of white respondents (28\%) felt that their health had deteriorated despite treatment at a clinic, while some coloured respondents (12\%) felt the same about hospital treatment. In the case of medical practitioners, $16 \%$ of coloured respondents, $13 \%$ of white respondents and $9 \%$ of black respondents reported that these services had had no effect on their health. As regards clinic services, $16 \%$ of responses from whites, $15 \%$ of those from blacks and $11 \%$ of those from coloureds indicated that their health had remained unchanged, while significant proportions of blacks (18\%), coloureds (18\%) and whites (15\%) reported similarly with respect to hospital treatment. If PRHC has no perceivable effect on health, it is likely that PRHC services and facilities will be less well utilised or not utilised at all (Van Vuuren 1992:139-140).

\section{SUMMARY AND CRITICAL COMMENTS}

As far as the accessibility of PRHC as manifested in the utilisation pattern of the group under investigation is concerned, the white population group made use of the services of medical practitioners, while the coloured population group used clinics as well as hospital services, and the black population group relied on hospital services. In all three groups, the initial point of contact in case of illness was the doctor. As far as payment for services was concerned, most whites are backed up by medical schemes, while coloureds and blacks, few of whom belonged to medical schemes, generally paid personally for medical services. Although there were some reservations, all three population groups viewed the cost of PRHC as reasonable. Concerning the distance of service points it appears that the black respondents experience the most problems in this regard. While white respondents reported that they were assisted quickly at PRHC points of service, coloured and black respondents felt that they had to wait a long time before being assisted. White and black respondents categorised the attitude of PRHC staff as generally friendly, but a high percentage of coloured respondents were of the opinion that hospital staff were unfriendly towards patients. With some reservations, respondents were satisfied with the PRHC services they had used. With the exception of the coloured group, respondents considered the times of PRHC services to be convenient. With regard to the perceived effect of PRHC on health, divergent opinions were voiced. In some cases an improvement in health was reported, while in other cases a deterioration was noted; yet others considered their state of health unchanged after making use of PRHC.

When the finer nuances of the above-mentioned utilisation pattern of PRHC are taken into account, it appears that, in comparison with white users, coloured and black users of PRHC are at a disadvantage in 
terms of the affordability and acceptability of health care and that, as a result of these inhibiting factors, PRHC is more accessible for some groups than for others. The inaccessibility of health care does not only affect the utilisation thereof, but also has an effect on the heaith care requirements which manifest themselves.

Critical consideration of the accessibility of PRHC in the three communities emphasises the following health care issues:

- Although utilisation patterns provide a handy measure for the identification of underprovision, overprovision or sufficient provision and accessibility, inaccessibility of PRHC services and facilities, according to Maaga (1992), health planners who have to make decisions about PRHC do not take enough cognisance of these patterns.

- The inequitable and fragmentary manner in which PRHC is offered or provided in the area under investigation affects the use of services and facilities to such an extent that health care is more accessible, acceptable and affordable for some people than for others. It is self-evident that people who cannot pay for PRHC have no access to such care and cannot thus make use of it. The same is true when services are inaccessible in terms of distance. The utilisation of PRHC also depends on factors such as the time taken in travelling to the doctor, the time waited before being able to consult the doctor, the consultation hours of the doctor, the geographical situation of the relevant point of service, etc.

- In addition, it appears that people make use of specific types of PRHC to provide for their health care requirements. The respective types of care - private practitioners, clinics and hospital facilities - are differentially utilised, in accordance with the group to which the user belongs and his/her financial position. On these grounds, the utilisation of health care services and facilities can definitely be categorised as inequitable.

- According to Aday, Andersen and Fleming (1980:26), the question of whether PRHC services and facilities are accessible also depends on the structural characteristics of the system of provision and on the nature of the requirements of potential users. This implies, for example, that a person who requires preventative services must have access to said services for it is unlikely that use of curative or hospital-based care will be made in this case. Viewed in this way, the supply, demand and utilisation components of the PRHC system are intimately related, and the utilisation component is indeed the product of the interaction of the supply and demand components of the PRHC system. Above all, it must be bome in mind that there are factors making PRHC services less accessible for some than for others. As Dutton says "Given the rapid evolution of current modes of health care delivery, information about the effects of different provider and system features on patterns of care is vital in assessing the changes presently occurring and in informing the choices which lie ahead" (1986:731). Therefore the issues raised on the accessibility of PRHC form part of a wide range of topics that needs to be considered by PRHC practitioners, health care planners and policy makers to enable all people to exercise their basic right to health care.

\section{NOTES}

1. This article has been adapted from Chapter 4 in the report: Professionele gesondheidsorg in drie gemeenskappe: Aanbod-, behoefte- en verbruikspatrone, done within and financial aid from the HSRC Co-operative Research Programme: Affordable Social Provision. This report (no BBS/ASS-36) is available from: HSRC Publications, $\mathrm{p} / \mathrm{b} \mathrm{X} 41$, Pretoria (ISBN 079691412 5).

2. Although the Population Registration Act has been repealed, these terms are used and the population groups are discussed separately in order to illustrate differential accessibility to and thus inequities in PRHC. Using these terms should not be construed as implying acceptance of the erstwhile stantory divisions.

\section{Greater Bloemfontein represents the} municipally delimited Bloemfontein area. Heidedal and Mangaung. At the time of the survey in October 1990, the total population of the Greater Bloemfontein area was 259 300. This total includes 102800 whites in the municipal area, 23900 coloureds in Heidedal and 132600 blacks in Mangaung. These figures do not include the approximately 16 000 residents of squatter areas at the time. These residents are now (1994) estimated at approximately 60000 . (Figures calculated by $R B$ van der Merwe, Department of Business Management, UOFS, according to Central Statistical Service Data, 1986)

4. Tables 2 to 11 refer to responses not to respondents.

\section{REFERENCES}

Aday, LA, Andersen, R \& Fleming, GV. 1980. Health care in the US. Equitable for whom? London: SAGE.

Anyinam, C. 1987. Availability, accessibility, acceptability and adaptability: four attributes of African ethno-medicine. Social Science and Medicine, 25(7):803-811.

BENATAR, SR. 1989. Apartheid and medical care. South African Medical Journal, 75:559.

COE, RM. 1978. Sociology of medicine. New York: McGraw-Hill

FOSU, GB. 1989. Access to health care in urban areas of developing societies. Journal of Health and Social Behaviour, 30:398-411.

MAAGA, MP. 1992. The problem of explaining health care services use behaviour - a critique. Pretoria: ASSA Conference, 28 June - 1 July

SAVAGE, M \& BENATAR, SR. 1990. An analysis of health and health services. In Schrire, R (ed). Critical choices for South Africa. An agenda for the 1990s. Cape Town: Oxford University: 147-167.

SENTRALE STATISTIEKDIENS. 1986 Bevolkingsensus 1985: ouderdom volgens ontwikkelingstreek, statistiese streek en distrik. Pretoria: Staatsdrukker (verslag no 02-85-02).

VAN RENSBURG, HCJ \& BENATAR, SR. 1993. The legacy of apartheid in health and health care. South African Journal of Sociology, 24(4):99-111.

VAN VUUREN, SJEJ. 1992. Professionele gesondheidsorg in drie gemeenskappe: ' $n$ kritiese analise van aanbod-, behoefte- en verbruikspatrone. Ongepubliseerde D Phil-proefskrif. Bloemfontein: Universiteit van die Oranje-Vrystaat.

VAN VUUREN, SJEJ, DE KLERK, GW \& VAN RENSBURG, HCJ. 1993. Professionele gesondheidsorg in drie gemeenskappe: Aanbod-, behoefte- en verbruikspatrone. Pretoria: RGN Koöperatiewe Navorsingsprogram: Bekostigbare Maatskaplike Voorsiening (verslag no BBS/ASS-36).

Dr S JE J van Vuuren

Sociolo gy Department

Uni versity of the Orange Free State

Prof G W de K lerk

Sociolo gy Department

Uni versity of the Orange Free State 